K. Ichihara

Nagoya Math. J.

Vol. 187 (2007), 75-90

\title{
LARGE DEVIATIONS FOR RADIAL RANDOM WALKS ON HOMOGENEOUS TREES
}

\author{
KANJI ICHIHARA
}

\begin{abstract}
Donsker-Varadhan's type large deviation will be discussed for the pinned motion of a radial random walk on a homogeneous tree. We shall prove that the rate function corresponding to the large deviation is associated with a new Markov chain constructed from the above random walk through a harmonic transform based on a positive principal eigenfunction for the generator of the random walk.
\end{abstract}

\section{§1. Introduction}

In [5] Donsker-Varadhan's type large deviation results have been obtained for the pinned motions of two classes of diffusion processes (Brownian motion on a hyperbolic plane and reversible, periodic diffusion processes on $\mathbb{R}^{n}$ ) which have a strong transience property in general. It has been shown that the corresponding rate functions are related to new diffusion processes defined through harmonic transforms based on positive principal eigenfunctions for the generators associated with the original processes. This type of large deviations seem to be of great importance for the study of Schrödinger kernels.

Analogous large deviations can be considered for Markov chains. In fact, Chiyonobu, Ichihara and Mituisi [1] have obtained large deviations for a class of periodic, reversible Markov chains on $\mathbb{Z}^{n}$. The main result in the paper can be extended to a class of reversible Markov chains on a discrete group of polynomial volume growth.

In this paper we shall discuss the above problem for random walks on a class of trees. There occurs a crucial difference between discrete groups of polynomial volume growth (euclidean type) and trees (hyperbolic type) where principal eigenfunctions play an important role. Thus another scheme is required.

Received June 2, 2005

Revised March 28, 2006, July 3, 2006.

2000 Mathematics Subject Classification: 60F10, 60J10. 
Let $\mathbb{T}^{q+1}(q \geq 2)$ be an infinite homogeneous tree of order $q+1$. Namely it is a connected graph without loops or cycles and each vertex has $(q+1)$ nearest neighbours. One characteristic feature of the tree is that for every pair of vertices $x, y$ there is a unique path connecting $x$ and $y$ with length $d(x, y)$, where $d(x, y)$ is the number of edges in the path and plays the role of a distance on $\mathbb{T}^{q+1}$.

Let $X_{n}$ be a random walk on $\mathbb{T}^{q+1}$. We are interested in an asymptotic behaviour of the Feynman-Kac type expectation:

$$
E^{P_{(0, x)}^{(n, y)}}\left\{\exp \left(-\sum_{k=0}^{n-1} m\left(X_{k}\right)\right)\right\} \quad \text { as } n \rightarrow \infty
$$

where $P_{(0, x)}^{(n, y)}$ denotes the probability law of the process $X$. pinned as $X_{0}=$ $x, X_{n}=y$ and $m(x)$ is a bounded function on $\mathbb{T}^{q+1}$. The first step to investigate such a behaviour is to establish large deviation results for the occupation time distribution of the pinned random walk.

From now on, we consider a random walk on $\mathbb{T}^{q+1}$ whose one step transition probability $p(x, y)$ satisfies the following conditions:

(A.1) The transition probability $p(x, y)$ depends only on the distance $d(x, y)$. $(\mathrm{A} .2) \inf \{p(x, y) \mid d(x, y) \leq 1\}>0$.

This type of random walk on a homogeneous tree has been treated in Sawyer [8]. He has discussed asymptotic behaviors for the radial part of the sample function and for the $n$-step transition probabilities. Some further results in this direction can be found in [6], [7], [9], [10] and references therein.

Define a difference operator $L$ for a function $u(x)$ on $\mathbb{T}^{q+1}$ by

$$
L u(x)=\sum_{y \in \mathbb{T}^{q+1}} p(x, y)(u(y)-u(x)) .
$$

Denote by $\mu_{0}$ the counting measure on $\mathbb{T}^{q+1}$. Then $L$ is a self-adjoint operator in the Hilbert space $L^{2}\left(\mathbb{T}^{q+1}, \mu_{0}\right)$.

Let $\mathcal{M}$ be the set of probability measures on $\mathbb{T}^{q+1}$ endowed with the weak topology. Let $\Omega_{x}$ be the space of all sequences $X_{0}, X_{1}, X_{2}, \ldots$ with $X_{0}=x$ and $X_{i} \in \mathbb{T}^{q+1}$. We have a probability measure on $\Omega_{x}$ induced by $p(\cdot, \cdot)$, which is denoted by $P_{x}$ and is called the random walk associated 
with the transition probability $\{p(x, y)\}$. For each $\omega \in \Omega_{x}$, each positive integer $n$ and a subset $A$ of $\mathbb{T}^{q+1}$, define

$$
L_{n}(\omega, A)=\frac{1}{n} \sum_{k=0}^{n-1} \chi_{A}\left(X_{k}(\omega)\right)
$$

and for a measurable subset $B \subseteq \mathcal{M}$,

$$
Q_{(0, x)}^{(n, y)}(B)=P_{(0, x)}^{(n, y)}\left(\omega ; L_{n}(\omega, \cdot) \in B\right)
$$

In this paper a large deviation principle for $Q_{(0, x)}^{(n, y)}$ will be investigated. In order to introduce a rate function appropriate for our formulation, we first discuss a generalized eigenvalue problem for the difference operator $L$ related to the random walk $\left(X_{n}, P_{x}\right)$.

Let $S_{k}(x)$ be the sphere centered at $x$ with radius $k \in \mathbb{N} \cup\{0\}$ i.e.

$$
S_{k}(x)=\left\{y ; d(x, y)=k, y \in \mathbb{T}^{q+1}\right\}
$$

Evidently, $\left|S_{0}(x)\right|=1$ and $\left|S_{k}(x)\right|=(q+1) q^{k-1}(k \geq 1)$.

Under the assumption (A.1), we may set

$$
p(x, y)=A(d)=A_{d}, \quad d=d(x, y)
$$

and

$$
P\left(d\left(X_{n+1}, X_{n}\right)=d \mid X_{n}=x\right)=A_{d}^{*}=q^{d-1}(q+1) A_{d}, \quad d>0
$$

with $A_{0}^{*}=A_{0}$. Note that the numbers $\left\{A_{d}\right\}$ satisfy a relation

$$
A_{0}+\sum_{d=1}^{\infty} q^{d-1}(q+1) A_{d}=1
$$

For a fixed point $x_{0} \in \mathbb{T}^{q+1}$, define

$$
u_{0}(x)=\left(1+\frac{q-1}{q+1} d\left(x_{0}, x\right)\right) q^{-d\left(x_{0}, x\right) / 2}, \quad x \in \mathbb{T}^{q+1}
$$

and

$$
\lambda_{0}=1-\sum_{d=0}^{\infty} q^{-d / 2}\left(1+\frac{q-1}{q+1} d\right) A_{d}^{*}
$$

It is easily verified that $\lambda_{0} \in(0,1)$. 
THEOREM 1. It holds that for $u_{0}(x)$ and $\lambda_{0}$ defined above,

$$
L u_{0}(x)+\lambda_{0} u_{0}(x)=0, \quad x \in \mathbb{T}^{q+1} .
$$

It should be remarked that the number $\lambda_{0}$ is the greatest lower bound of the $L^{2}$-spectrum of the difference operator $-L$, see [10] and that $u_{0}(x)$ is a corresponding (generalized) eigenfunction. This theorem is an immediate consequence of the following lemma which is implicit in Proposition 2.4, Chapter II, [4].

Lemma 1. For $x \in \mathbb{T}^{q+1}, m \in \mathbb{N}$

$$
\sum_{y: d(x, y)=m} u_{0}(y)=q^{m / 2-1}(q+1)\left(1+\frac{q-1}{q+1} m\right) u_{0}(x) .
$$

Making use of the function $u_{0}(x)$ above, a new transition probability function $p^{0}(x, y)$ is defined by

$$
p^{0}(x, y)=\frac{p(x, y) u_{0}(y)}{\left(1-\lambda_{0}\right) u_{0}(x)}, \quad x, y \in \mathbb{T}^{q+1} .
$$

Let $\left(X_{n}^{0}, P_{x}^{0}\right)$ be the Markov chain on $\mathbb{T}^{q+1}$ induced by $\left\{p^{0}(x, y)\right\}$. From the definition of $p^{0}(x, y)$, the Markov chain $\left(X_{n}^{0}, P_{x}^{0}\right)$ is reversible with respect to $u_{0}^{2}$ i.e. $u_{0}(x)^{2} p^{0}(x, y)=u_{0}(y)^{2} p^{0}(y, x)$. Denote by $p_{n}^{0}(x, y)$ the $n$ step transition probability of the Markov chain $X_{n}^{0}$. Then $p_{n}^{0}(x, y)$ satisfies $p_{n}^{0}(x, y)=\frac{u_{0}(y)}{\left(1-\lambda_{0}\right)^{n} u_{0}(x)} p_{n}(x, y)$.

Set

$$
\pi^{0} u(x)=\sum_{y \in \mathbb{T}^{q+1}} p^{0}(x, y) u(y)
$$

Denote by $\mathcal{U}$ the set of positive functions $u$ on $\mathbb{T}^{q+1}$ for each of which there exist constants $a$ and $b$ such that $0<a \leq u(x) \leq b<\infty$ for all $x \in \mathbb{T}^{q+1}$.

We now introduce a functional on $\mathcal{M}$ which plays the role of a rate function in our case.

$$
I_{0}(\mu)=-\inf _{u \in \mathcal{U}} \sum_{x \in \mathbb{T}^{q+1}} \log \left(\frac{\pi^{0} u}{u}\right)(x) \mu(x), \quad \mu \in \mathcal{M} .
$$

Note that $I_{0}(\mu)<\infty$ for any $\mu \in \mathcal{M}$. In fact, under the assumption (A.2), we have that for any $x \in \mathbb{T}^{q+1}$

$$
\pi^{0} u(x) \geq \frac{p(x, x)}{1-\lambda_{0}} u(x)=\frac{p\left(x_{0}, x_{0}\right)}{1-\lambda_{0}} u(x) .
$$


Hence

$$
-I_{0}(\mu)=\inf _{u \in \mathcal{U}} \sum_{x \in \mathbb{T}^{q+1}} \log \left(\frac{\pi^{0} u}{u}\right)(x) \mu(x) \geq \log \left(\frac{p\left(x_{0}, x_{0}\right)}{1-\lambda_{0}}\right)>-\infty,
$$

which implies that $I_{0}(\mu)<\infty$ for any $\mu \in \mathcal{M}$.

Our main results are the following.

TheOREM 2. (i) For any compact $C \subseteq \mathcal{M}$,

$$
\limsup _{n \rightarrow \infty} \frac{1}{n} \log Q_{(0, x)}^{(n, y)}(C) \leq-\inf _{\mu \in C} I_{0}(\mu) \text {. }
$$

(ii) For any open $G \subseteq \mathcal{M}$,

$$
\liminf _{n \rightarrow \infty} \frac{1}{n} \log Q_{(0, x)}^{(n, y)}(G) \geq-\inf _{\mu \in G} I_{0}(\mu) .
$$

TheOREm 3. For any bounded function $m(x)$ on $\mathbb{T}^{q+1}$,

$$
\begin{aligned}
& \lim _{n \rightarrow \infty} \frac{1}{n} \log E^{P_{(0, x)}^{(n, y)}}\left\{\exp \left(-\sum_{k=0}^{n-1} m\left(X_{k}\right)\right)\right\} \\
& =-\inf _{\mu \in \mathcal{M}}\left\{I_{0}(\mu)+\sum_{x \in \mathbb{T}^{q+1}} m(x) \mu(x)\right\} .
\end{aligned}
$$

\section{§2. Proof of Theorem 2: Upper bound}

We shall first prove the upper bound in Theorem 2 .

Set for a function $u \in \mathcal{U}$,

$$
V(x)=\pi^{0} u(x), \quad W(x)=\log \left(\frac{V(x)}{u(x)}\right) .
$$

From the definition of the pinned process,

$$
\begin{aligned}
& E^{P_{(0, x)}^{(n, y)}}\left\{u\left(X_{n-1}\right) \exp \left(-\sum_{k=0}^{n-1} W\left(X_{k}\right)\right)\right\} \\
& =\frac{1}{p_{n}(x, y)} E^{P_{x}}\left\{u\left(X_{n-1}\right) \exp \left(-\sum_{k=0}^{n-1} W\left(X_{k}\right)\right) p\left(X_{n-1}, y\right)\right\} \\
& =\frac{1}{p_{n}(x, y)} \sum_{x_{1}, \ldots, x_{n-1} \in \mathbb{T}^{q+1}} u\left(x_{n-1}\right) \exp \left(-\sum_{k=0}^{n-1} W\left(x_{k}\right)\right) \\
& \quad \times p\left(x_{n-1}, y\right) p\left(x, x_{1}\right) p\left(x_{1}, x_{2}\right) \cdots p\left(x_{n-2}, x_{n-1}\right),
\end{aligned}
$$


inserting $p(x, y)=\left(1-\lambda_{0}\right) u_{0}(x) p^{0}(x, y) / u_{0}(y)$ into the above,

$$
\begin{aligned}
& =\frac{\left(1-\lambda_{0}\right)^{n-1} u_{0}(x)}{p_{n}(x, y)} \sum_{x_{1}, \ldots, x_{n-1} \in \mathbb{T}^{q+1}} u\left(x_{n-1}\right) \exp \left(-\sum_{k=0}^{n-1} W\left(x_{k}\right)\right) \\
& \quad \times \frac{p\left(x_{n-1}, y\right)}{u_{0}\left(x_{n-1}\right)} p^{0}\left(x, x_{1}\right) p^{0}\left(x_{1}, x_{2}\right) \cdots p^{0}\left(x_{n-2}, x_{n-1}\right) \\
& =\frac{\left(1-\lambda_{0}\right)^{n-1} u_{0}(x)}{p_{n}(x, y)} E^{P_{x}^{0}}\left\{u\left(X_{n-1}^{0}\right) \exp \left(-\sum_{k=0}^{n-1} W\left(X_{k}^{0}\right)\right) \frac{p\left(X_{n-1}^{0}, y\right)}{u_{0}\left(X_{n-1}^{0}\right)}\right\} .
\end{aligned}
$$

Making use of the boundedness of $p(z, y) / u_{0}(z)$ in $z$ for a fixed $y$ and an estimate of $p_{n}(x, y)$ for a large $n$, see Theorem 19.30 in [10], page 215, we have

$$
\begin{aligned}
& E^{P_{(0, x)}^{(n, y)}}\left\{u\left(X_{n-1}\right) \exp \left(-\sum_{k=0}^{n-1} W\left(X_{k}\right)\right)\right\} \\
& \leq C_{1} \frac{\left(1-\lambda_{0}\right)^{n-1} u_{0}(x)}{p_{n}(x, y)} E^{P_{x}^{0}}\left\{u\left(X_{n-1}^{0}\right) \exp \left(-\sum_{k=0}^{n-1} W\left(X_{k}^{0}\right)\right)\right\} \\
& \leq C_{2} n^{3 / 2} u_{0}(y) e^{C_{3} d(x, y)^{2} / n} E^{P_{x}^{0}}\left\{u\left(X_{n-1}^{0}\right) \exp \left(-\sum_{k=0}^{n-1} W\left(X_{k}^{0}\right)\right)\right\}
\end{aligned}
$$

following arguments in [2], page 8

$$
=C_{2} n^{3 / 2} u_{0}(y) e^{C_{3} d(x, y)^{2} / n} u(x) .
$$

Thus we have obtained

$$
E^{P_{(0, x)}^{(n, y)}}\left\{\exp \left(-\sum_{k=0}^{n-1} W\left(X_{k}\right)\right)\right\} \leq C_{2} \frac{n^{3 / 2} u_{0}(y) e^{C_{3} d(x, y)^{2} / n} u(x)}{\inf _{x \in \mathbb{T}^{q+1}} u(x)} .
$$

The definition of $Q_{(0, x)}^{(n, y)}$ implies

$$
\begin{aligned}
& E^{P_{(0, x)}^{(n, y)}}\left\{\exp \left(-\sum_{k=0}^{n-1} W\left(X_{k}\right)\right)\right\} \\
& =E^{P_{(0, x)}^{(n, y)}}\left\{\exp \left(-n \sum_{z \in \mathbb{T}^{q+1}} W(z) L_{n}(\omega,\{z\})\right)\right\} \\
& =E^{Q_{(0, x)}^{(n, y)}}\left\{\exp \left(-n \sum_{z \in \mathbb{T}^{q+1}} W(z) \mu(z)\right)\right\} .
\end{aligned}
$$


Consequently it follows that for any measurable subset $C$ of $\mathcal{M}$

$$
\begin{aligned}
& E^{Q_{(0, x)}^{(n, y)}}\left[\exp \left(-n \sum_{z \in \mathbb{T}^{q+1}} W(z) \mu(z)\right)\right] \\
& \geq Q_{(0, x)}^{(n, y)}(C) \exp \left(-n \sup _{\mu \in C} \sum_{z \in \mathbb{T}^{q+1}} W(z) \mu(z)\right) .
\end{aligned}
$$

Combining (4) and (5), we obtain

$$
Q_{(0, x)}^{(n, y)}(C) \leq C_{2} \frac{n^{3 / 2} u_{0}(y) e^{C_{3} d(x, y)^{2} / n} u(x)}{\inf _{x \in \mathbb{T}^{q+1}} u(x)} \cdot \exp \left(n \sup _{\mu \in C} \sum_{z \in \mathbb{T}^{q+1}} W(z) \mu(z)\right) .
$$

Since $u$ is an arbitrary element of $\mathcal{U}$, we have

$$
\limsup _{n \rightarrow \infty} \frac{1}{n} \log Q_{(0, x)}^{(n, y)}(C) \leq \inf _{u \in \mathcal{U}} \sup _{\mu \in C} \sum_{z \in \mathbb{T}^{q+1}} \log \left(\frac{\pi^{0} u}{u}\right)(z) \mu(z) .
$$

The upper bound in Theorem 2 now follows from the same reasoning as in [2], page 9 .

\section{§3. Proof of Theorem 2: Lower bound}

We need some auxiliary results from [3].

Let $\mathcal{M}_{\mathbb{T}^{q+1} \times \mathbb{T}^{q+1}}$ be the space of all probability measures on $\mathbb{T}^{q+1} \times \mathbb{T}^{q+1}$ and let $\mathcal{M}_{\alpha, \beta}$ be the set of all $\lambda \in \mathcal{M}_{\mathbb{T}^{q+1} \times \mathbb{T}^{q+1}}$ with first marginal $\alpha$ and second marginal $\beta$. $\mathcal{U}_{1}$ denotes the set of functions $u(x, y)$ on $\mathbb{T}^{q+1} \times \mathbb{T}^{q+1}$ for each of which there are constants $a$ and $b$ such that $0<a \leq u(x, y) \leq b<\infty$ on $\mathbb{T}^{q+1} \times \mathbb{T}^{q+1}$. For any $\lambda \in \mathcal{M}_{\mathbb{T}^{q+1} \times \mathbb{T}^{q+1}}$, we define

$$
\begin{aligned}
\bar{I}(\lambda)=-\inf _{u \in \mathcal{U}_{1}}\left\{\log \left\{\sum_{\mathbb{T}^{q+1} \times \mathbb{T}^{q+1}} u(x, y) \lambda_{0}(x, y)\right\}\right. \\
\left.-\sum_{\mathbb{T}^{q+1} \times \mathbb{T}^{q+1}} \log u(x, y) \cdot \lambda(x, y)\right\}
\end{aligned}
$$

where $\lambda_{0}(x, y)=\gamma(x) p^{0}(x, y), \gamma$ being the first marginal of $\lambda$.

Let a probability measure $\mu \in \mathcal{M}$ have full support. As is remarked after the introduction of the rate function $I_{0}(\lambda), I_{0}(\lambda)<\infty$ for any $\lambda \in \mathcal{M}$. Applying Theorem 2.1 in [3], we see that

$$
I_{0}(\mu)=\inf _{\lambda \in \mathcal{M}_{\mu, \mu}} \bar{I}(\lambda) .
$$


Since $\bar{I}(\lambda)$ is lower semicontinuous and since $\mathcal{M}_{\mu, \mu}$ is compact, the infimum in (7) is actually attained. Denote the element of $\mathcal{M}_{\mu, \mu}$ where this infimum is attained by $\bar{\lambda}$, i.e., $\bar{I}(\bar{\lambda})=I_{0}(\mu)$. We write $\bar{\lambda}(x, y)=\mu(x) \bar{p}^{0}(x, y)$, for all $(x, y) \in \mathbb{T}^{q+1} \times \mathbb{T}^{q+1}$. Note that $\bar{p}^{0}(x, y)$ becomes the transition probability of a new Markov process on $\mathbb{T}^{q+1}$. The probability measure $\mu$ is an invariant measure for $\bar{p}^{0}$, since $\mu$ is not only a first marginal for $\bar{\lambda}$ but also a second marginal. Denote by $\bar{P}^{0}$ the probability for the stationary discrete parameter Markov process having $\bar{p}^{0}(x, y)$ as its transition probability and the invariant measure $\mu$ as initial distribution. Denote by $\bar{P}_{x}^{0}$ the corresponding probabilities for this process starting at $x$. In the proof of lower bound in Theorem 2, we need the fact that the stationary process $\bar{P}^{0}$ is ergodic. In order to show this, we proceed as follows. From the definition of $I_{0}(\mu)$, there exists a sequence $\left\{u_{n}\right\}$ in $\mathcal{U}$ such that

$$
\lim _{n \rightarrow \infty} \sum_{x \in \mathbb{T}^{q+1}} \log \left(\frac{\pi^{0} u_{n}}{u_{n}}\right)(x) \mu(x)=-I_{0}(\mu) .
$$

Let

$$
\lambda_{n}(x, y)=\mu(x) \frac{u_{n}(y)}{\left(\pi^{0} u_{n}\right)(x)} p^{0}(x, y)
$$

and let $\bar{\lambda}$ be as above.

Since $I_{0}(\mu)<\infty$, Lemma 2.4 in [3] implies that

$$
\lim _{n \rightarrow \infty}\left\|\lambda_{n}-\bar{\lambda}\right\|=0
$$

where the norm is the variation norm. Taking the discrete situation into account, this gives that for any $(x, y) \in \mathbb{T}^{q+1} \times \mathbb{T}^{q+1}$

$$
\lim _{n \rightarrow \infty} \lambda_{n}(x, y)=\bar{\lambda}(x, y)
$$

From this, we get

Lemma 2. Under the assumption that $\operatorname{supp}(\mu)=\mathbb{T}^{q+1}$

$$
h(x, y):=\lim _{n \rightarrow \infty} \frac{u_{n}(y)}{\pi^{0} u_{n}(x)}
$$

exists and is strictly positive for all $(x, y) \in \mathbb{T}^{q+1} \times \mathbb{T}^{q+1}$. 
Proof. First note that (A.1) together with the hypotheses guarantees the existence of $\lim _{n \rightarrow \infty} u_{n}(y) / \pi^{0} u_{n}(x)$ for all $x$ and $y$ satisfying $d(x, y) \leq 1$.

Since $I_{0}(\mu)<\infty$ and $\pi^{0} u_{n}(x) / u_{n}(x)$ is uniformly bounded below by a positive constant $p^{0}\left(x_{0}, x_{0}\right) /\left(1-\lambda_{0}\right)$, we have

$$
\lim _{n \rightarrow \infty} \frac{\pi^{0} u_{n}(x)}{u_{n}(x)}<\infty, \quad x \in \mathbb{T}^{q+1}
$$

For $x$ and $y$ such as $d(x, y) \geq 2$, choose a sequence $x_{k}, k=0,1, \ldots, n_{0}$ such that

$$
\begin{gathered}
x_{0}=x, x_{1}, \ldots, x_{n_{0}}=y \\
d\left(x_{k}, x_{k+1}\right)=1, \quad k=0,1, \ldots, n_{0}-1 .
\end{gathered}
$$

Then we have

$$
\begin{aligned}
\frac{u_{n}(y)}{\pi^{0} u_{n}(x)}=\frac{u_{n}\left(x_{1}\right)}{\pi^{0} u_{n}\left(x_{0}\right)} \frac{\pi^{0} u_{n}\left(x_{1}\right)}{u_{n}\left(x_{1}\right)} \frac{u_{n}\left(x_{2}\right)}{\pi^{0} u_{n}\left(x_{1}\right)} \cdots \\
\quad \ldots \frac{u_{n}\left(x_{n_{0}-1}\right)}{\pi^{0} u_{n}\left(x_{n_{0}-2}\right)} \frac{\pi^{0} u_{n}\left(x_{n_{0}-1}\right)}{u_{n}\left(x_{n_{0}-1}\right)} \frac{u_{n}\left(x_{n_{0}}\right)}{\pi^{0} u_{n}\left(x_{n_{0}-1}\right)} .
\end{aligned}
$$

Hence letting $n \rightarrow \infty$, from the above we get the existence of $\lim _{n \rightarrow \infty}$ $u_{n}(y) / \pi^{0} u_{n}(x)$ for all $x$ and $y$ in $\mathbb{T}^{q+1}$. Applying Lemma 2.5 in [3], we get that there exist two functions $a(x)$ and $b(y)$ such as

$$
h(x, y):=\lim _{n \rightarrow \infty} \frac{u_{n}(y)}{\pi^{0} u_{n}(x)}=\frac{a(x)}{b(y)}
$$

where $0 \leq a(x)<\infty$ and $0<b(y) \leq \infty$. Since $h(x, x)>0$,

$$
0<a(x), b(y)<\infty
$$

for any $x, y$. This completes the proof of Lemma 2.

We shall now complete the proof of the lower bound in Theorem 2 . Assume $\mu \in G$ has full support on $\mathbb{T}^{q+1}$. Denote by $\bar{\lambda}$ an element of $\mathcal{M}_{\mu, \mu}$ for which $\bar{I}(\bar{\lambda})=I_{0}(\mu)$ as before. Define $\bar{p}^{0}(x, y)$ by $\bar{\lambda}(x, y)=\mu(x) \bar{p}^{0}(x, y)$. Since

$$
\bar{\lambda}(x, y)=\mu(x) \frac{a(x)}{b(y)} p^{0}(x, y),
$$


it follows from Lemma 2.1 in [2] that

$$
I_{0}(\mu)=\sum_{\mathbb{T}^{q+1} \times \mathbb{T}^{q+1}} \log (a(x) / b(y)) \bar{\lambda}(x, y)
$$

It is possible to find a sphere $S(\mu, \epsilon)$ with center $\mu$ and radius $\epsilon>0$ with respect to the Prokhorov metric such that $S(\mu, \epsilon) \subseteq G$. Set

$$
F_{n, \epsilon}=\left\{\omega=\left(x_{j}\right)_{j \geq 0} \in \Omega_{x} ; \frac{1}{n-1} \sum_{j=1}^{n-1} \log \left(a\left(x_{j-1}\right) / b\left(x_{j}\right)\right) \leq I_{0}(\mu)+\epsilon\right\}
$$

Let $\bar{P}^{0}$ and $\bar{P}_{x}^{0}$ be the probabilities introduced before. The $\bar{P}^{0}$ process is a stationary process and, by Lemma 2 , is a mixing process. Hence by virtue of the ergodic theorem, we have

$$
\begin{gathered}
\bar{P}_{x}^{0}\left(\omega ; L_{n}(\omega, \cdot) \text { converges to } \mu \text { as } n \rightarrow \infty\right)=1 \\
\bar{P}_{x}^{0}\left(\omega ; \lim _{n \rightarrow \infty} \frac{1}{n-1} \sum_{j=1}^{n-1} \log \left(a\left(\bar{X}_{j-1}^{0}\right) / b\left(\bar{X}_{j}^{0}\right)\right)\right. \\
\left.=\sum_{\mathbb{T}^{q+1} \times \mathbb{T}^{q+1}} \log (a(x) / b(y)) \bar{\lambda}(x, y)\right)=1
\end{gathered}
$$

Making use of the same harmonic transform as in the proof of the upper bound, we get

$$
\begin{gathered}
P_{(0, x)}^{(n, y)}\left(\omega ; L_{n}(\omega, \cdot) \in G\right) \geq P_{(0, x)}^{(n, y)}\left(\omega ; L_{n}(\omega, \cdot) \in S(\mu, \epsilon)\right) \\
=\frac{\left(1-\lambda_{0}\right)^{n-1} u_{0}(x)}{p_{n}(x, y)} E^{P_{x}^{0}}\left\{\frac{p\left(X_{n-1}^{0}, y\right)}{u_{0}\left(X_{n-1}^{0}\right)} ; L_{n} \in S(\mu, \epsilon)\right\},
\end{gathered}
$$

which is expressed by means of the Markovian measure $\left(\bar{X}_{n}^{0}, \bar{P}_{x}^{0}\right)$ as

$$
\begin{aligned}
& \frac{\left(1-\lambda_{0}\right)^{n-1} u_{0}(x)}{p_{n}(x, y)} \\
& \quad \times E^{\bar{P}_{x}^{0}}\left\{\frac{p\left(\bar{X}_{n-1}^{0}, y\right)}{u_{0}\left(\bar{X}_{n-1}^{0}\right)} \exp \left(-\sum_{j=1}^{n} \log h\left(\bar{X}_{j-1}^{0}, \bar{X}_{j}^{0}\right)\right) ; L_{n} \in S(\mu, \epsilon)\right\}
\end{aligned}
$$




$$
\begin{aligned}
& \geq \frac{\left(1-\lambda_{0}\right)^{n-1} u_{0}(x)}{p_{n}(x, y)} \\
& \quad \times E^{\bar{P}_{x}^{0}}\left\{\frac{p\left(\bar{X}_{n-1}^{0}, y\right)}{u_{0}\left(\bar{X}_{n-1}^{0}\right)} \exp \left(-(n-1)\left[I_{0}(\mu)+\epsilon\right]\right) ; L_{n} \in S(\mu, \epsilon)\right\} \\
& \geq \frac{\left(1-\lambda_{0}\right)^{n-1} u_{0}(x)}{p_{n}(x, y)} \exp \left(-(n-1)\left[I_{0}(\mu)+\epsilon\right]\right) \\
& \quad \times E^{\bar{P}_{x}^{0}}\left\{\frac{p\left(\bar{X}_{n-1}^{0}, y\right)}{u_{0}\left(\bar{X}_{n-1}^{0}\right)} ;\left\{L_{n} \in S(\mu, \epsilon)\right\} \cap F_{n, \epsilon}\right\} .
\end{aligned}
$$

Combining the estimate for $p_{n}(x, y)$ and the boundedness of $p(\cdot, y) / u_{0}(\cdot)$ with the ergodicity of the process $\bar{X}_{n}^{0}$, we get by letting $n \rightarrow \infty$ and $\epsilon \rightarrow 0$ that

$$
\lim _{n \rightarrow \infty} \frac{1}{n} \log Q_{(0, x)}^{(n, y)}(G) \geq-I_{0}(\mu)
$$

Let $\mathcal{M}_{0}$ be the set of probability measures on $\mathbb{T}^{q+1}$ with full support. Then it is easy to prove that under our assumptions

$$
\inf _{\mu \in G} I_{0}(\mu)=\inf _{\mu \in G \cap \mathcal{M}_{0}} I_{0}(\mu)
$$

for any open subset $G \subseteq \mathcal{M}$.

This together with (8) completes the proof of the lower bound.

\section{$\S 4$. Proof of Theorem 3}

In this section we shall discuss an asymptotic behavior of the FeynmanKac type functional associated with the walk.

Let $L^{2}\left(\mathbb{T}^{q+1}, u_{0}^{2}\right)$ be the set of real valued functions on $\mathbb{T}^{q+1}$ which are square integrable with respect to the measure $u_{0}^{2}$. Denote by $\langle f, g\rangle$ the inner product on $L^{2}\left(\mathbb{T}^{q+1}, u_{0}^{2}\right)$ i.e. $\langle f, g\rangle=\sum_{x \in \mathbb{T}^{q+1}} f(x) g(x) u_{0}(x)^{2}$.

Let $h$ be a bounded function on $\mathbb{T}^{q+1}$. Introduce an operator $A_{h}$ for a bounded function $f$ on $\mathbb{T}^{q+1}$ by

$$
A_{h} f(x)=e^{-h(x) / 2} \sum_{y \in \mathbb{T}^{q+1}} p^{0}(x, y) e^{-h(y) / 2} f(y)=e^{-h(x) / 2} \pi^{0}\left(e^{-h / 2} f\right)(x) .
$$

It can be easily seen that the operator $A_{h}$ defines a bounded operator on $L^{2}\left(\mathbb{T}^{q+1}, u_{0}^{2}\right)$ and that

$$
\left\langle A_{h} f, g\right\rangle=\left\langle f, A_{h} g\right\rangle, \quad f, g \in L^{2}\left(\mathbb{T}^{q+1}, u_{0}^{2}\right),
$$


holds. Hence $A_{h}$ is self-adjoint on $L^{2}\left(\mathbb{T}^{q+1}, u_{0}^{2}\right)$.

Let $u$ be in $\mathcal{U}$. We follow the same notations $V(x)=\pi_{0} u(x)$ and $W(x)=\log (V(x) / u(x))$ as in the proof of Theorem 2 .

On the other hand, by the same reasoning as in [2], page 8 ,

$$
E^{P_{x}^{0}}\left\{u\left(X_{n-1}^{0}\right) \exp \left\{-\left[W\left(X_{0}^{0}\right)+W\left(X_{1}^{0}\right)+\cdots+W\left(X_{n-1}^{0}\right)\right]\right\}\right\}=u(x) .
$$

Thus

(9) $E^{P_{x}^{0}}\left\{\exp \left\{-\left[W\left(X_{0}^{0}\right)+W\left(X_{1}^{0}\right)+\cdots+W\left(X_{n-1}^{0}\right)\right]\right\}\right\} \leq \frac{u(x)}{\inf _{y \in \mathbb{T}^{q+1}} u(y)}$.

This implies the left hand-side in (9) is uniformly bounded for a fixed function $u$ in $\mathcal{U}$.

It is easy to see that

$$
\begin{aligned}
& A_{W}^{n} f(x)=e^{W(x) / 2} E^{P_{x}^{0}}\left\{\exp \left\{-\left[W\left(X_{0}^{0}\right)+W\left(X_{1}^{0}\right)+\cdots+W\left(X_{n}^{0}\right)\right]\right\}\right. \\
& \left.\times e^{W\left(X_{n}^{0}\right) / 2} f\left(X_{n}^{0}\right)\right\} .
\end{aligned}
$$

In particular, $A_{W}^{n} 1$ is uniformly bounded on $\mathbb{T}^{q+1}$ by a constant depending only on $u$.

We have for $f \in L^{2}\left(\mathbb{T}^{q+1}\right)$,

$$
\left\langle A_{W}^{n} f, A_{W}^{n} f\right\rangle=\sum_{x \in \mathbb{T}^{q+1}}\left(A_{W}^{n} f\right)^{2}(x) u_{0}(x)^{2},
$$

the Schwartz inequality applied to $\left(A_{W}^{n} f\right)^{2}$ together with the uniform boundedness of $A_{W} 1$ and the symmetry of the operator $A_{W}$ implies,

$$
\begin{aligned}
& \leq \sum_{x \in \mathbb{T}^{q+1}} A_{W}^{n}\left(f^{2}\right) A_{W}^{n} 1(x) u_{0}(x)^{2} \leq C \sum_{x \in \mathbb{T}^{q+1}} A_{W}^{n}\left(f^{2}\right) u_{0}(x)^{2} \\
& =C \sum_{x \in \mathbb{T}^{q+1}} A_{W}^{n} 1(x) f(x)^{2} u_{0}(x)^{2} \leq C^{2} \sum_{x \in \mathbb{T}^{q+1}} f(x)^{2} u_{0}(x)^{2}=C^{2}\langle f, f\rangle .
\end{aligned}
$$

Hence we get $\left\|A_{W}^{n}\right\|_{L^{2}\left(\mathbb{T}^{q+1}, u_{0}^{2}\right)} \leq C, n=1,2, \ldots$ Denoting by $\lambda_{W}$ the upper bound of the $L^{2}$ spectrum for $A_{W}$, the above implies that $\lambda_{W}$ is less than or equal to 1 i.e.

$$
\lambda_{W}=\sup _{f:\langle f, f\rangle=1}\left\langle A_{W} f, f\right\rangle \leq 1 .
$$


Thus we have for a nonnegative function $f$ with $\langle f, f\rangle=1$,

$$
\begin{aligned}
0 \geq \log \left\langle A_{W} f, f\right\rangle & \\
= & \log \left(\sum_{x, y \in \mathbb{T}^{q}+1} e^{-W(x) / 2} p^{0}(x, y) e^{-W(y) / 2} f(y) f(x) u_{0}^{2}(x)\right) \\
= & \log \left(\sum_{x, y \in \mathbb{T}^{q+1}} e^{\left\{-\frac{W(x)}{2}+\frac{m(x)}{2}\right\}+\left\{-\frac{W(y)}{2}+\frac{m(y)}{2}\right\}}\right. \\
& \left.\quad \times \frac{e^{-m(x) / 2} p^{0}(x, y) e^{-m(y) / 2} f(x) f(y) u_{0}(x)^{2}}{\left\langle A_{m} f, f\right\rangle}\right)+\log \left\langle A_{m} f, f\right\rangle,
\end{aligned}
$$

by virtue of Jensen's inequality

$$
\begin{aligned}
& \geq \sum_{x, y \in \mathbb{T}^{q+1}}\left(\left\{-\frac{W(x)}{2}+\frac{m(x)}{2}\right\}+\left\{-\frac{W(y)}{2}+\frac{m(y)}{2}\right\}\right) \\
& \quad \times \frac{e^{-m(x) / 2} p^{0}(x, y) e^{-m(y) / 2} f(x) f(y) u_{0}(x)^{2}}{\left\langle A_{m} f, f\right\rangle}+\log \left\langle A_{m} f, f\right\rangle \\
& =\sum_{x \in \mathbb{T}^{q+1}}(-W(x)+m(x)) \frac{\left(A_{m} f\right)(x) f(x) u_{0}(x)^{2}}{\left\langle A_{m} f, f\right\rangle}+\log \left\langle A_{m} f, f\right\rangle .
\end{aligned}
$$

Introduce a probability measure on $\mathbb{T}^{q+1}$ defined by

$$
\mu_{m, f}(x):=\frac{\left(A_{m} f\right)(x) f(x) u_{0}(x)^{2}}{\left\langle A_{m} f, f\right\rangle}
$$

Then the above inequality implies

$$
\sum_{x \in \mathbb{T}^{q+1}} \log \left(\frac{\pi^{0} u}{u}\right)(x) \mu_{m, f}(x)-\sum_{x \in \mathbb{T}^{q+1}} m(x) \mu_{m, f}(x) \geq \log \left\langle A_{m} f, f\right\rangle, \quad u \in \mathcal{U}
$$

taking the infimum of the left hand-side over $\mathcal{U}$, we get

$$
-I_{0}\left(\mu_{m, f}\right)-\sum_{x \in \mathbb{T}^{q+1}} m(x) \mu_{m, f}(x) \geq \log \left\langle A_{m} f, f\right\rangle
$$


Noting that $\mu_{m, f}$ is an element of $\mathcal{M}$, we finally see

$$
\begin{aligned}
& \sup _{\mu \in \mathcal{M}}\left\{-I_{0}(\mu)-\sum_{x \in \mathbb{T}^{q+1}} m(x) \mu(x)\right\} \\
& \geq \sup _{\substack{\langle f, f\rangle=1 \\
f \geq 0}}\left\{-I_{0}\left(\mu_{m, f}\right)-\sum_{x \in \mathbb{T}^{q+1}} m(x) \mu_{m, f}(x)\right\} \\
& \geq \sup _{\substack{\langle f, f\rangle=1 \\
f \geq 0}} \log \left\langle A_{m} f, f\right\rangle=\log \lambda_{m} .
\end{aligned}
$$

Thus we have obtained

$$
-\inf _{\mu \in \mathcal{M}}\left\{I_{0}(\mu)+\sum_{x \in \mathbb{T}^{q+1}} m(x) \mu(x)\right\} \geq \log \lambda_{m} .
$$

Combining this with Theorem 2, (ii), we get

$$
\begin{aligned}
& \liminf _{n \rightarrow \infty} \frac{1}{n} \log E^{P_{(0, x)}^{(n, y)}}\left\{\exp \left(-\sum_{k=0}^{n-1} m\left(X_{k}\right)\right)\right\} \\
& \geq-\inf _{\mu \in \mathcal{M}}\left\{I_{0}(\mu)+\sum_{x \in \mathbb{T}^{q+1}} m(x) \mu(x)\right\} \geq \log \lambda_{m} .
\end{aligned}
$$

Consequently, in order to prove Theorem 3, it suffices to show

$$
\limsup _{n \rightarrow \infty} \frac{1}{n} \log E^{P_{(0, x)}^{(n, y)}}\left\{\exp \left(-\sum_{k=0}^{n-1} m\left(X_{k}\right)\right)\right\} \leq \log \lambda_{m}
$$

From the definition of the pinned process

$$
\begin{aligned}
& E_{(0, x)}^{P_{(n, y)}^{(n)}}\left\{\exp \left(-\sum_{k=0}^{n-1} m\left(X_{k}\right)\right)\right\} \\
& =\frac{1}{p_{n}(x, y)} E^{P_{x}}\left\{\exp \left(-\sum_{k=0}^{n-1} m\left(X_{k}\right)\right) p\left(X_{n-1}, y\right)\right\}
\end{aligned}
$$


making use of the same harmonic transform as in the proof of Theorem 2 (upper bound),

$$
\begin{aligned}
& =\frac{\left(1-\lambda_{0}\right)^{n-1} u_{0}(x)}{p_{n}(x, y)} E^{P_{x}^{0}}\left\{\exp \left(-\sum_{k=0}^{n-1} m\left(X_{k}^{0}\right)\right) \frac{p\left(X_{n-1}^{0}, y\right)}{u_{0}\left(X_{n-1}^{0}\right)}\right\} \\
& =\frac{\left(1-\lambda_{0}\right)^{n-1} u_{0}(x)}{p_{n}(x, y)} e^{-m(x) / 2} A_{m}^{n-1}\left(e^{-m(\cdot) / 2} \frac{p(\cdot, y)}{u_{0}(\cdot)}\right)(x) .
\end{aligned}
$$

Applying the Schwarz inequality, we get from the above

$$
\begin{aligned}
& A_{m}^{n-1}\left(e^{-m(\cdot) / 2} \frac{p(\cdot, y)}{u_{0}(\cdot)}\right)(x)=A_{m} A_{m}^{n-2}\left(e^{-m(\cdot) / 2} \frac{p(\cdot, y)}{u_{0}(\cdot)}\right)(x) \\
& \leq e^{-m(x) / 2} \sqrt{\sum_{z \in \mathbb{T}^{q+1}} \frac{p^{0}(x, z)^{2} e^{-m(z)}}{u_{0}(z)^{2}}} \\
& \times \sqrt{\sum_{z \in \mathbb{T}^{q+1}}\left[A_{m}^{n-2}\left(e^{-m(\cdot) / 2} \frac{p(\cdot, y)}{u_{0}(\cdot)}\right)(z)\right]^{2} u_{0}(z)^{2}} \\
& \leq C_{x, y} \sqrt{\left\langle A_{m}^{n-2}\left(e^{-m(\cdot) / 2} \frac{p(\cdot, y)}{u_{0}(\cdot)}\right), A_{m}^{n-2}\left(e^{-m(\cdot) / 2} \frac{p(\cdot, y)}{u_{0}(\cdot)}\right)\right\rangle}
\end{aligned}
$$

where $C_{x, y}$ is a positive constant depending only on $x, y$ and $m$. Since $e^{-m(\cdot) / 2} p(\cdot, y) / u_{0}(\cdot)$ is square integrable with respect to the measure $u_{0}^{2}$, we get

$$
\begin{aligned}
& A_{m}^{n-1}\left(e^{-m(\cdot) / 2} \frac{p(\cdot, y)}{u_{0}(\cdot)}\right)(x) \\
& \leq C_{x, y} \lambda_{m}^{n-2} \sqrt{\left\langle e^{-m(\cdot) / 2} \frac{p(\cdot, y)}{u_{0}(\cdot)}, e^{-m(\cdot) / 2} \frac{p(\cdot, y)}{u_{0}(\cdot)}\right\rangle} .
\end{aligned}
$$

This implies that the inequality (15) is valid.

Acknowledgements. The author is grateful to an anonymous referee for useful comments, suggestions and calling his attention to additional references on random walks and harmonic analysis on trees. 


\section{REFERENCES}

[1] T. Chiyonobu, K. Ichihara and H. Mituisi, Large Deviation for Periodic Markov Process on Square Lattice, J. Math. Sci. Univ. Tokyo, 13 (2006), 525-544.

[2] M. D. Donsker and S. R. S. Varadhan, Asymptotic evaluation of certain Markov process expectations for large time, I, Comm. Pure Appl. Math., 28 (1975), 1-47.

[3] M. D. Donsker and S. R. S. Varadhan, Asymptotic evaluation of certain Markov process expectations for large time, III, Comm. Pure Appl. Math., 29 (1976), 389461.

[4] A. Figà-Talamanca and C. Nebbia, Harmonic Analysis and Representation Theory for Groups Acting on Homogeneous Trees, London Mathematical Society Lecture Note Series, vol. 162.

[5] K. Ichihara, Large deviation for pinned covering diffusion, Bull. Sci. math., 125 (2001), 529-551.

[6] S. Lalley, Finite range random walks on free groups and homogeneous trees, Ann. Prob., 21 (1993), 2087-2130.

[7] F. Ledrappier, Some asymptotic properties of random walks on free groups, Topics in probability and Lie groups: boundary theory, CRM Proc. Lecture Notes, vol. 28, Amer. Math. Soc., Providence, RI, 2001, pp. 117-152.

[8] S. Sawyer, Isotropic random walks in a tree, Zeit. Wahr. verw. Gebiete, 42 (1978), 279-292.

[9] S. Sawyer and T. Steger, The rate of escape for anisotropic random walks in a tree, Prob. Th. Rel. Fields, 76 (1987), 207-230.

[10] W. Woess, Random walks on infinite graphs and groups, Cambridge Tracts in Mathematics, vol. 138, Cambridge University Press.

Department of Mathematics

Faculty of Engineering Science

Kansai University

Suita, Osaka 564-8680

Japan

kchihara@ipcku.kansai-u.ac.jp 